\section{Lunar Occultation of a Radio Source}

At present only a small number of radio sources have beon satisfactorily identified with photographic objects. The important task of establishing further identifications faces a serious difficulty because of the uncertainty in the measured positions of the radio sources. This uncertainty usually amounts to several minutes of arc, and it is only for a few intense sources that the error has been reduced to less than one minute of arc.

It has been suggested by several authors, for example by Link and Neuzil ${ }^{1}$, that observations should be made of the occultation of radio sources by the Moon, and this technique has already been used to study some intense sources of large angular diameter ${ }^{2-5}$. It is the purpose of this communication to direct attention to the high precision with which source positions can be measured by observing lunar occultations with equipment which can track the Moon continuously. The results of a preliminary test of the technique on a radio source are described. here.

On December 8, 1960, the 250-ft. radio telescope at Jodrell Bank was used for observing the occultation by the Moon of the radio source $3 C 212$ (ref. 6). The measurements were made at a frequency of $237 \mathrm{Mc}$./s. using a receiver with a band-width of $2 \mathrm{Mc}$./s. and a post-detector time constant of 4 sec. The observations were carried out by recording the output of the receiver with the telescope in automatic motion following the centre of the Moon. The beam-width of the aerial was about $1 \cdot 2^{\circ}$ between half-power points and therefore covered an area considerably larger than the Moon's disk.

The occultation was observed as a sharp fall in the received power as the source was covered by the Moon, followed later by a sharp rise as the source reappeared. The measured times at which these events occurred are :

Time of disappearance $08 \mathrm{~h} .03 \mathrm{~m} .52 \cdot 5 \mathrm{~s} . \pm 5 \mathrm{~s}$. U.T. Time of reappearance $08 \mathrm{~h} .40 \mathrm{~m} .49 \mathrm{~s} . \pm 5 \mathrm{~s}$.

These times correspond to the passage of the source across the limb of the Moon and they have been derived from the records on the assumption that the angular size of the source is small compared with the diffraction pattern of the Moon's limb.

H.M. Nautical Almanac Office has kindly cal. culated the celestial co-ordinates of the source from the observed times which define two possible positions of the source, one on either side of the centre of the Moon on a line perpendicular to its direction of motion. In principle this ambiguity can be resolved by observations of a subsequent occultation; however, in the present case, the position given in the Cambridge $3 C$ Catalogue is sufficiently precise to allow one of the two possible positions to be chosen. The calculated mean place of the source for 1950.0 is given bolow together with the data from the Cambridge $3 C$ Catalogue.

$$
\begin{aligned}
& \text { Position computed from oceultation (1950.0) } \\
& \begin{array}{l}
\text { Pource } 3 C \text { 212: R.A. 08h. 55m. } 55 \cdot 7 \mathrm{~s}_{*} \pm 0.3 \mathrm{~s} . \\
\text { Dec. 14. } 21^{\prime} 27^{\prime \prime} \pm 2^{\prime \prime}
\end{array} \\
& \text { Catalogue data (6) } \\
& \text { Source 3C 212: R.A. 08h. } 56 \mathrm{~m} .00 \mathrm{~s} . \pm 5 \mathrm{~s} \text {. } \\
& \text { Dec, } 14^{\circ} 25^{\prime} \pm 8^{\prime} \text { 的. }
\end{aligned}
$$

The quoted errors in the computed position have been estimated only from the errors in measurement of the time of immersion and emersion, and neglect any possible refraction in a lunar ionosphere or the effect of irregularities in the Moon's limb. The high degree of accuracy achieved is due to the circumstances of the occultation. The closest approach of the source to the centre of the Moon was about 12 minutes of arc, and under these conditions an error of one minute in the duration of the occultation introduces an error of only $12^{\prime \prime}$ of are in the declination. If the source had passed close to the centre of the Moon the accuracy would have been considerably reduced, as in this case a similar error in duration would have produced a declination error of about three minutes of are.

The measurement reported here probably represents the most accurate determination yet made of the position of a radio source. The method used has the great advantage that it is not subject to uncertainties introduced by refraction in the Earth's troposphere or ionosphere. It is also free from the effects of confusion, as the solid angle subtended by the Moon's diffraction pattern is only of the order $1 / 100$ sq. degree. A significant source of error is likely to lie in the assumption that the angular diameter of the source is negligible. However, a preliminary comparison of the observed occultation curves with the theoretical diffraction pattern shows that the diameter of the source must be less than or equal to eight seconds of arc, and hence the resultant error in declination will not be greater than approximately four seconds of are. It may therefore be concluded that, taking into account all sources of error, the estimated position of the source is probably accurate to within about five seconds of arc.

The work described here was carried out as part of a programme designed to measure accurate positions of a number of radio sources by the method of lunar occultations. A detailed description of the method together with an analysis of all the data so far obtained will be published later.

I wish to thank Sir Bernard Lovell and Prof. $R$. Hanbury Brown for their interest in the work, and Mrs. Flora McBain Sadler of H.M. Nautical Almanac Office for the calculation of the position of the source.

University of Manchester, C. HAZARD *

Nuffield Radio Astronomy Laboratories, Jodrell Bank.

* Present address: School of Physics, University of Sydney, New South Wales.

${ }^{1}$ Link, F., and Neuzil, L., Bull. Astro. Insts. Csl., 5, 112 (1954).

'Elsmore, B., and Whitfield, C. R., Nature, 176, 447 (1955).

${ }^{3}$ Boischot, A., Blum, E. J., Ginat, M., and le Roux, E., C.R. Acad. Sci., Paris., 242, 1849 (1956).

${ }^{4}$ Rishbeth, H., Austral. J. Phys., 9, 494 (1956).

Rishbeth, H., and Little, A. G., The Observatory, 77, 897 (1957).

- Edge, D. O., Shakeshaft, J. R., McAdam, W. B., Baldwin, J. E., and Archer, S., Mem. Roy. Astro. Soc., 68, 37 (1959).

\section{PHYSICS}

\section{Thermoelectric Power of Single Crystals of Gallium}

As part of a programme to investigate the anisotropy of non-cubic erystals, the thermoelectric power of single crystals of the orthorhombic metal gallium has been examined. The aim of the programme is the explanation of the large magnitude of the anisotropies of the physical properties of gallium.

The single crystals were prepared from gallium of at least 99.99 per cent purity, and they were grown in the form of cylindrical rods encased in a glass tube. The length of the specimens was $10-13 \mathrm{~cm}$. and their 